\title{
Structural Joining of Ceramics Nanoparticles: Development of Photonic Crystals for Terahertz Wave Control by Using Micro Stereolithography ${ }^{\dagger}$
}

\author{
Soshu Kirihara \\ Smart Processing Research Center, Joining and \\ Welding Research Institute, Osaka Universit ${ }^{1}$
}

\begin{abstract}
Structural joining is new concept of materials processing to create novel functional materials with special patterns and morphologies. Nanometer sized ceramics particles are bound continuously with resin materials solidified by laser beam irradiations to form micrometer order structures exactly. In this paper, fabrication processes of alumina photonic crystals with a periodic diamond structure will be introduced. Periodic arrangements of dielectric constants can control electromagnetic waves in terahertz frequency ranges through spatial wave diffractions. Three dimensional dielectric lattices were designed by using graphical application of a computer aided designing (CAD) software, and acrylic diamond structures with alumina nanoparticles dispersion were formed by using micro stereolithography ( $\mu$-STL) of a computer aided manufacturing (CAD) system. Fabricated precursors were dewaxed and sintered in the air to obtained full ceramics photonic crystals. The terahertz wave properties were measured by terahertz time domain spectroscopy (TDS) device. A complete photonic band gap to reflect the terahertz wave perfectly was observed, and showed good agreement with a theoretical simulation of plane wave expansion (PWE) method. Moreover, localization of the terahertz wave were observed in point or plane defects introduced into the diamond photonic crystals trough an electromagnetic field analysis of transmission line modeling (TLM) method.
\end{abstract}

Keywords: Phtonic Crystal, Band Gap, Dielectric Material, Terahertz Wave, Stereolithography

\section{Introduction}

Periodically arranged structures of dielectric media are called photonic crystals ${ }^{1-4)}$. They have photonic band gaps in which no electromagnetic wave can propagate. If the periodicity is changed locally by introducing a defect, localized modes appear in the band $g \mathrm{p}^{5-8)}$. Such localization function of electromagnetic waves can be applied to various devices, for example resonators, waveguides, and antennas. Three dimensional photonic crystals with a diamond structure are regarded as the ideal photonic crystal since they can prohibit the propagation of electromagnetic waves for any directions in the band $\operatorname{gap}^{9,10)}$. However, due to the complex structure, they

\footnotetext{
Accepted: August $25^{\text {th }}, 2009$

1 11-1 Mihogaoka Iaraki, 567-0047 Osaka, Japan

TEL: 81-6-6879-8693

FAX: 81-6-6879-8693

E-mail: kirihara@jwri.osaka-u.ac.jp
}

are difficult to fabricate. In our previous investigations, we have succeeded in fabricating micrometer order diamond structures by using stereolithography method of a computer aided design and manufacturing $(\mathrm{CAD} / \mathrm{CAM})$ processes ${ }^{11-16)}$. Photo sensitive resin slurry including with nanometer sized ceramic particles was solidified continuously through laser beam scanning to create dielectric lattice structures accurately. A complete photonic band gap formation in the terahertz wave frequency range was reported. Subsequently, structural modifications of the diamond lattice structures to control the terahertz wave propagations were investigated by using the $\mathrm{CAD} / \mathrm{CAM}$ process practically ${ }^{17-21)}$. In near future industries, electromagnetic waves in a terahertz frequency range with micrometer order wavelength will be expected to apply for various types of novel sensors which can detect gun powders, drugs, bacteria in foods, micro cracks in electric devices, cancer cells in human skin and other physical, chemical and living events ${ }^{21-27)}$. 
In this paper, the novel stereolithography process to fat ${ }^{2}$ ricate the micro diamond photonic crystals by using the ceramic slurry with the nanoparticles will be introduced. And, the resonation and localization properties of the terahert $z$ waves into various types of the structural defects introduced according to theoretical electromagnetic simulations will be demonstrated.

\section{Photonic Band Gap Formation}

Photonic crystals composed of dielectric lattices form band gaps for electromagnetic waves. These artificial crystals can totally reflect light or microwave at a wavelength comparable to the lattice spacings by Bragg deflection as shown in Fig. 1. Two different standing waves oscillating in the air and dielectric matrix form higher and lower frequency bands in the first and second Brillouin zones, respectively. The band gap width can be controlled by varying structure, filling ratio, and dielectric constant of the lattice. Structural modifications by introducing defects or varying the lattice spacing can control the propagation of light or microwaves. The band diagram of the photonic crystal along symmetry lines in the Brillouin zone is drawn theoretically. The Maxwell's equations (1) and (2) can be solved by means of plane wave propagation (PWE) method ${ }^{28)}$, where $\omega$ and c denote frequency and light velocity, respectively. Electronic and magnetic field $\mathrm{E} \omega(\mathrm{r})$ and $\mathrm{H} \omega(\mathrm{r})$ are described with the following plane wave equations (3) and (4), respectively. The periodic arrangement of dielectric constant $\varepsilon$ (r) can be obtained as equation (5) form the crystal structure. $\mathrm{G}$ and $\mathrm{k}$ are reciprocal vector and wave vector, respectively.

$$
\begin{aligned}
& {\left[\nabla \times\left(\frac{1}{\varepsilon(\mathrm{r})}\right) \nabla \times\right] \mathbf{H}_{\omega}(\mathrm{r})=\left(\frac{\omega}{\mathrm{c}}\right)^{2} \mathbf{H}_{\omega}(\mathrm{r}) \cdots(1)} \\
& {\left[\frac{1}{\varepsilon(\mathrm{r})} \nabla \times \nabla \times\right] \mathbf{E}_{\omega}(\mathrm{r})=\left(\frac{\omega}{\mathrm{c}}\right)^{2} \mathbf{E}_{\omega}(\mathrm{r}) \cdots(2)}
\end{aligned}
$$

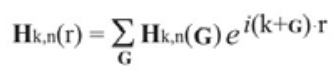

$$
\begin{aligned}
& \mathbf{E}_{\mathrm{k}, \mathrm{n}(\mathrm{r})}=\sum_{\mathbf{G}} \mathbf{E}_{\mathrm{k}, \mathrm{n}}(\mathbf{G}) e^{i(\mathrm{k}+\mathbf{G}) \mathrm{r}} \\
& \frac{1}{\varepsilon(\mathrm{r})}=\sum_{\mathbf{G}} \frac{1}{\varepsilon(\mathbf{G})} e^{i \mathbf{G} \cdot \mathrm{r}}
\end{aligned}
$$

\section{Applications of Photonic Crystals}

Fig. 2 shows expected applications of photonic crystal for light and electromagnetic wave control in various wavelength ranges ${ }^{29)}$. Air guides formed in a photonic crystal with nanometer order will be used as the light wave circuit in the perfect reflective structure. When a light emitting diode is placed in an air cavity formed in a photonic crystal, an efficient laser emission can be enhanced due to the high coherent resonance in the micro cavity. While, millimeter order periodic structures can control microwaves effectively. Directional antennas and filters composed of photonic crystals can be applied to millimeter wave radar devices for intelligent traffic system (ITS) and wireless communication system. The perfect reflection of millimeter wave by photonic crystal will be useful for barriers to prevent wave interference. Terahertz waves with micrometer order wavelength are expected to apply for various types of sensors to detect other physical, chemical and living events. The micrometer order photonic crystals can applied for the terahertz wave cavities, filters and antennas.

\section{Geometry of Artificial Crystals}

Typical photonic crystal structures were shown in
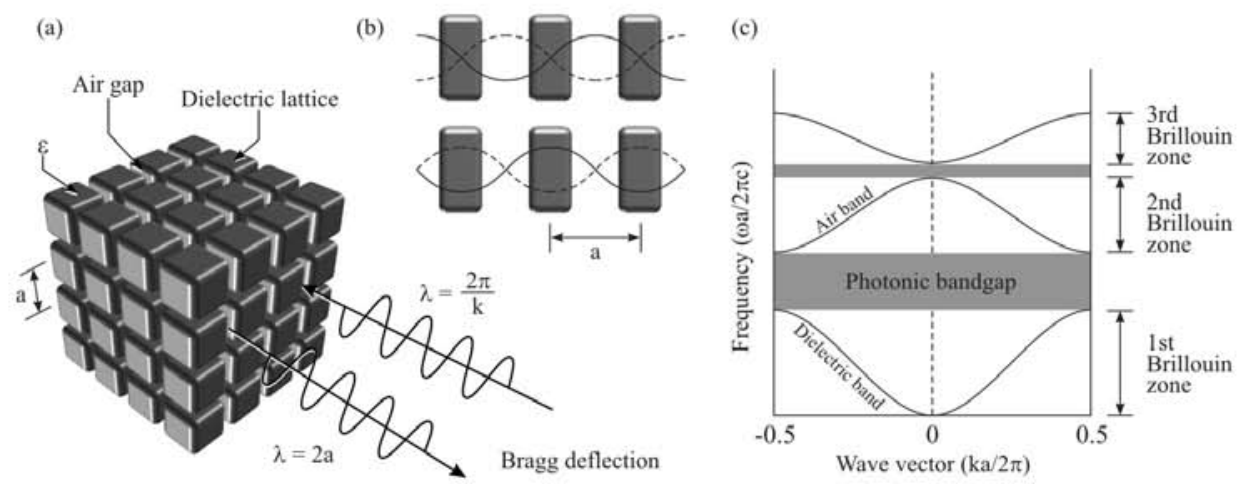

Fig. 1 Principles of photnic band gap formations in periodic arrangements of dielectric materials. Two different standing waves with higher and lower frequencies are formed in a photonic crystal, and a forbidden gap is formed between these frequencies. 


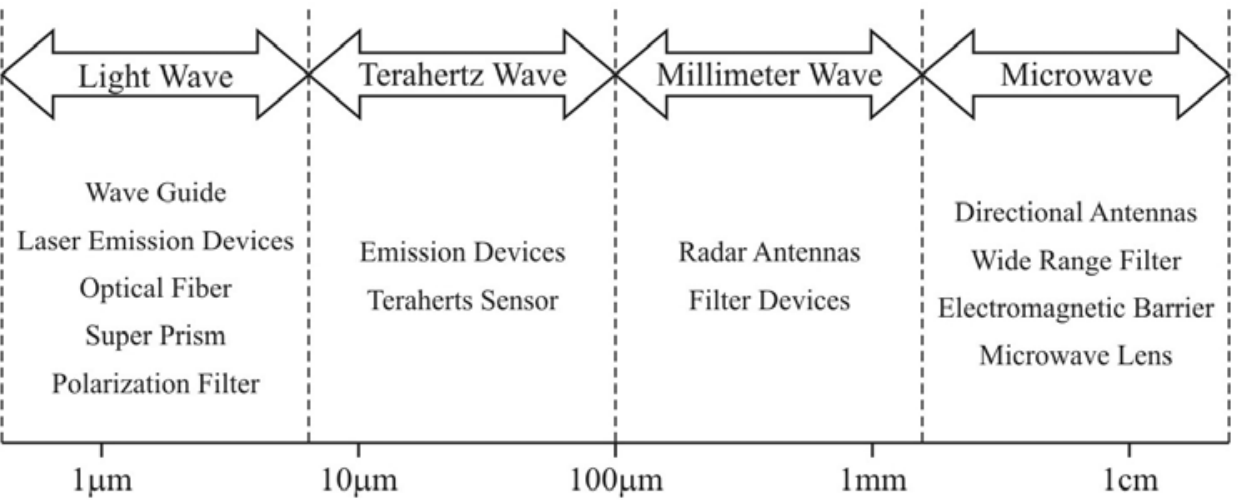

Electromagnetic Wavelength / Lattice Constant of Photonic Crystal

Fig. 2 Expected applications of the photonic crystal in various electromagnetic wavelengths.

Fig. 3. A woodpile structure (a) with simple structure of stacked rods can form the perfect photonic band gap. Photonic crystals composed of GaAs or InP were fabricated by using semiconductor process techniques $^{30)}$. A light wave circuit (b) in the periodic structure of arranged AlGaAs pins is processed by using electron beam lithography and etching techniques $^{31)}$. A layered structure (c) composed of $\mathrm{Si}$ and $\mathrm{SiO}_{2}$ with the different dielectric constants realize light wave polarization and super prism effects ${ }^{32}$. These layers are stacked by using self-organized growing in alternate spattering and etching. An inverse opal structure (d) is composed of air spheres with $\mathrm{FCC}$ structure in $\mathrm{TiO}_{2}, \mathrm{Si}, \mathrm{Ge}$ or $\mathrm{CdS}$ matrix ${ }^{33)}$. At first, polystyrene spheres are arranged by using self-organization in colloidal solutions. Then, the slurry of these dielectric media is infiltrated into the periodic structure and sintered. The optical fiber (e) with photonic crystal structure can guide light efficiently along the central core ${ }^{34)}$. Silica fibers and glass capillaries were bundled by wire drawing at high temperature. Diamond type photonic crystals (f) composed of $\mathrm{TiO}_{2}, \mathrm{SiO}_{2}$ or $\mathrm{Al}_{2} \mathrm{O}_{3}$ can fabricated by using stereolithography and successive sintering process. The wider perfect band gap is obtained in microwave and terahertz wave frequency ranges.

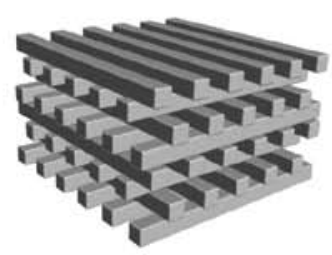

(a)

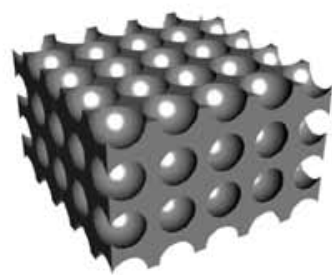

(d)

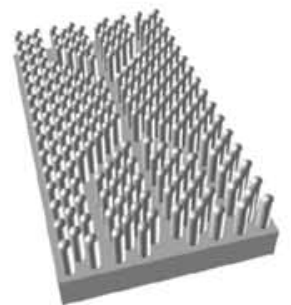

(b)

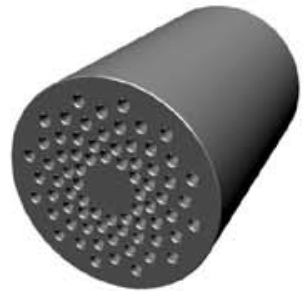

(e)

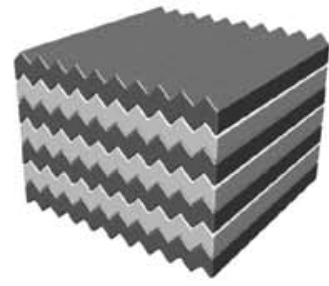

(c)

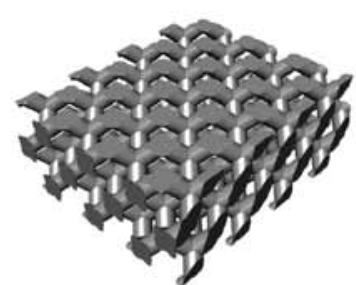

$(1)$

Fig. 3 Typical periodic structures of the photonic crystals with woodpile structure (a), patterned substrate (b), stacked layer (c), inverse opal structure (d), bundled fiber (e), and diamond structure (f). 
resin paste was fed with controlled air pressure. It was spread uniformly by moving a knife edge. The thickness of each layer was controlled to $10 \mu \mathrm{m}$. A two dimensional pattern was formed by illuminating visible laser of $405 \mathrm{~nm}$ in wavelength on the resin surface. The high resolution has been achieved by using a digital micro-mirror device (DMD) and an objective lens. Fig. 5 shows a schematic illustration of the micro stereolithography system. The DMD is an optical element assembled by mirrors of $14 \mu \mathrm{m}$ in edge length. The tilting of each tiny mirror can be controlled according to the two dimensional cross sectional data by using a computer. The three dimensional structure was built by stacking these micro patterns layer by layer. In order to avoid deformation and cracking during dewaxing, careful investigation for the dewaxing process is required. The precursors with diamond structures were heated at various temperatures from $100^{\circ} \mathrm{C}$ to $600^{\circ} \mathrm{C}$ while the heating rate was $1.0^{\circ} \mathrm{C} / \mathrm{min}$. The dewaxing process was observed in respect to the weight and color changes. Nanometer sized alumina particles could be sintered at 1500 ${ }^{\circ} \mathrm{C}$. The heating rate was $8.0^{\circ} \mathrm{C} / \mathrm{min}$. The density of the sintered sample was measured by the Archimedes method. The microstructure of the lattices was observed by using scanning electron microscopy (SEM).

\section{Measurement of Electromagnetic Wave}

In recent years, terahert $z$ waves have received extensive attentions and investigations since they have a lot of interesting and applicable features in various fields such as materials, communication, medicine, and biology. It is possible to detect gun powders and ceramic blades hidden in bags, clothes,

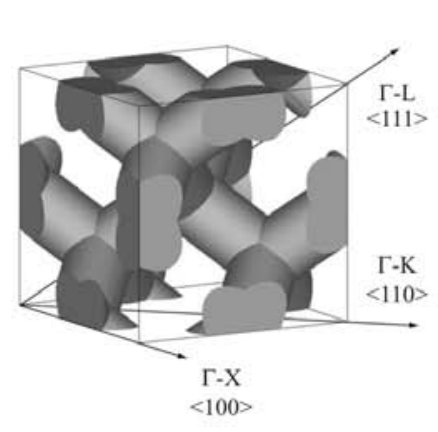

(a)

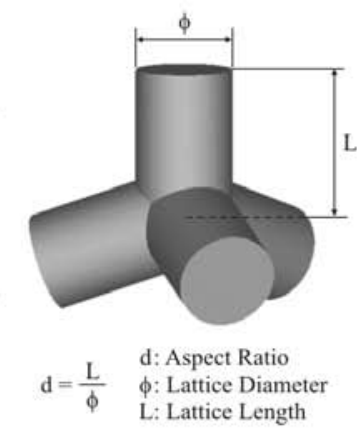

(b)

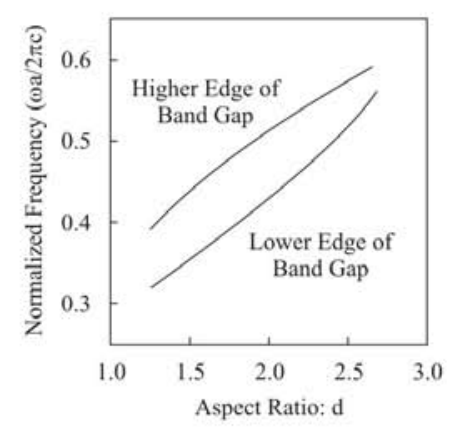

(c)

Fig. 4 A CAD image of a unit cell of diamond structure (a), definition of aspect ratio (b), and band gap width as a function of aspect ratio (c). 


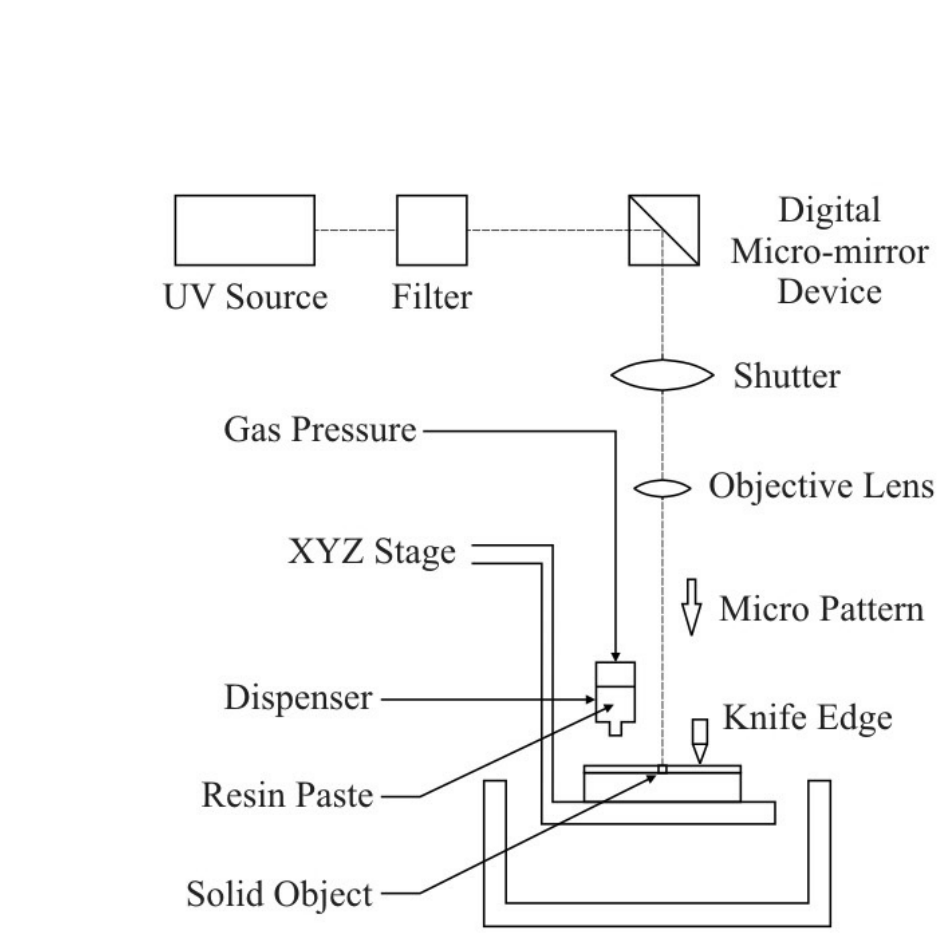

Fig. 5 A schematically illustrated free forming system of a micro stereolithography machine by using computer aided design and manufacturing (CAD/CAM) processes. (D-MEC Co. Ltd., Japan, SI-C 1000, http://www. d-mec.co.jp).

and envelopes by using terahertz waves since they can penetrate plastic, paper, and clothes without radiation damage to living bodies ${ }^{4)}$. It is also possible to identify toxic drugs because they have spectral fingerprints or absorption spectra ${ }^{5}$. Moreover, they can distinguish cancerous areas from healthy areas due to the different absorption rates ${ }^{6}$. A terahertz wave attenuation of transmission amplitudes through the diamond photonic crystals were measured by using a terahertz time domain spectrometer (TDS) apparatus (Advanced Infrared Spectroscopy Co. Ltd., Japan, Pulse-IRS 1000). Fig. 6 shows the schematic illustration of the measurement system. Femto second laser beams were irradiated into a micro emission antenna formed on a semiconductor substrate to generate the terahert $z$ wave pulses. The terahert $z$ waves were transmitted trough the micro patterned samples perpendicularly. The dielectric constant of the bulk samples were measured through a phase shift counting. Diffraction and resonation behaviors in the dielectric pattern were calculated theoretically by using a transmission line modeling (TLM) simulator (Flomerics, UK, Microstripes Ver. 7.5) of a finite difference time domain (FDTD) method.

\section{Alumina Photonic Crystals}

An alumina dispersed resin precursor fabricated by the micro stereolithography is shown in Fig. 7. The lattice constant of the formed diamond structure was

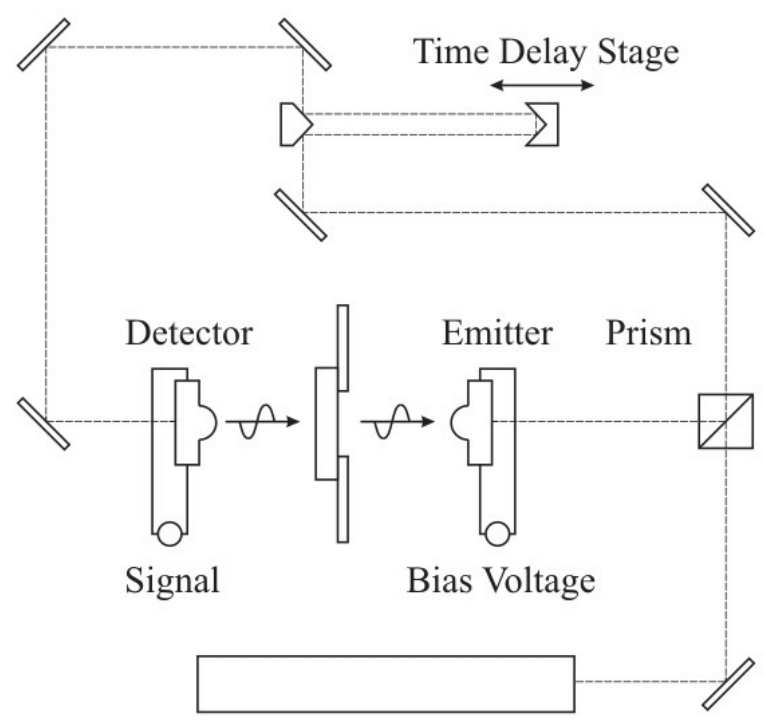

Femto Second Laser Source

Fig. 6 The schematically illustrated measuring system of a terahertz wave analyzer by using a time domain spectroscopic (TDS) detect method (Advanced Infrared Spectroscopy, Co. Ltd. Japan, J-Spec 2001, http://www. aispec.com).

(a)

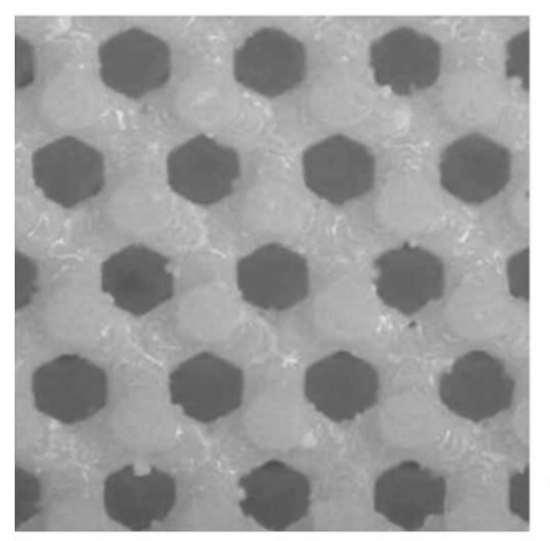

$\overline{250 \mu \mathrm{m}}$

(b)

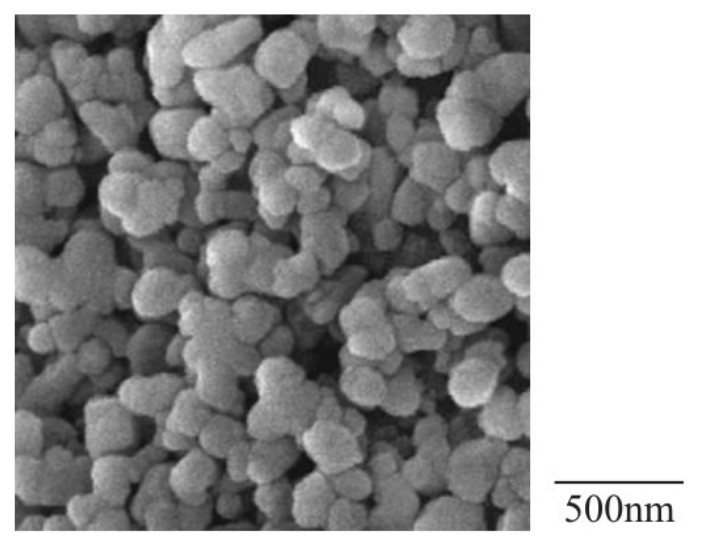

Fig. 7 The photonic crystal with the diamond structure composed of acrylic lattices with nano alumina particles dispersion formed by the micro stereolithography. 
500 m. TRe spatial resolution was approximately a $58 \%$ The weight and color changes as a function of temiperature are shown in Fig. 8. The sample color changed into black at $400^{\circ} \mathrm{C}$ due to carbonizing of resin. It became white at $600^{\circ} \mathrm{C}$ suggesting burning out of resin. Thus, the dewaxing process is considered to start at $200^{\circ} \mathrm{C}$ and complete at $600^{\circ} \mathrm{C}$. The dewaxing temperature was optimized to be $600^{\circ} \mathrm{C}$. Through the dewaxing and sintering processes, ceramic diamond structures were successfully obtained. Fig. 9 shows (111), (100) and (110) planes of the sintered diamond structure composed of the micrometer order alumina lattice. The lattice constant was measured as $375 \mu \mathrm{m}$. The deformation and cracking were not observed. The linear shrinkage on the horizontal axis was 23.8 $\%$ and that on the vertical axis was $24.6 \%$. It is possible to obtain the uniform shrinkage by designing an appropriate elongated structure in the vertical direction for compensation to the gravity effect. The relative density reached $97.5 \%$. Dense alumina microstructure was formed, and the average grain size was approximately $2 \mu \mathrm{m}$. The measured dielectric constant of the lattice was about 10 .

\section{Terahertz Wave Spectroscopy}

The terahertz wave attenuation of the transmission amplitude through the alumina diamond structure for $\Gamma-\mathrm{L}<111>, \Gamma-\mathrm{X}<100>$ and $\Gamma-\mathrm{K}<110>$ crystal direction is shown in Fig. 10. The forbidden gap is formed at the frequency rage of $0.32-0.49,0.35-0.53$

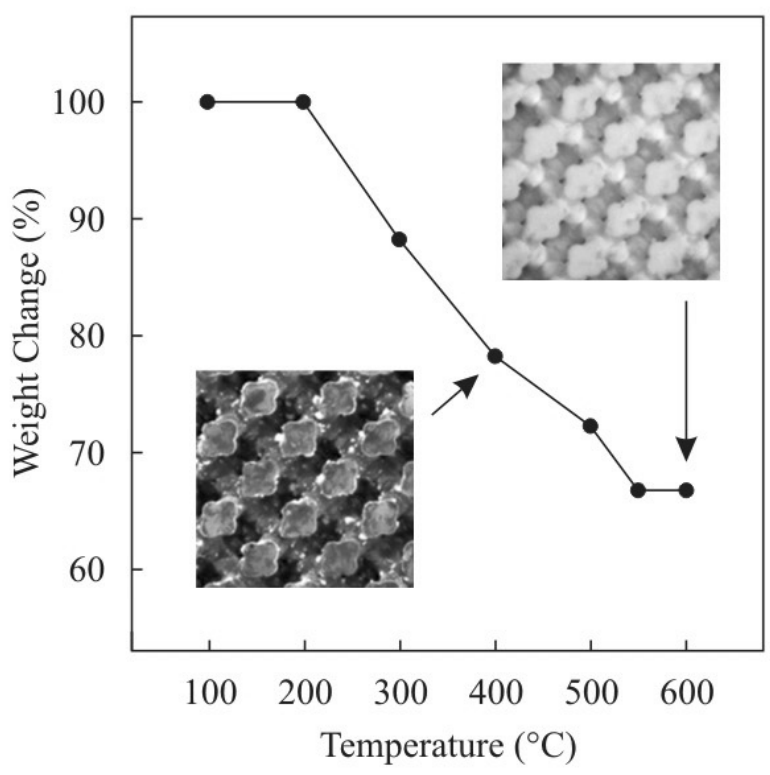

Fig. 8 The weight change as a function of temperature and the lattice color after dewaxing at 400 and $600^{\circ} \mathrm{C}$ in heating temperatures. and $0.35-0.52 \mathrm{THz}$ in transmission spectra for $\Gamma-\mathrm{L}$ $<111>, \Gamma-\mathrm{X}<100>$ and $\Gamma-\mathrm{K}<110>$ directions, respectively. A common band gap was observed in every direction at the frequency range from 0.35 to 0.49 $\mathrm{THz}$, where the electromagnetic wave cannot transmit through the crystal and is totally reflected in all directions. The measured band gap frequencies were compared with calculation results by the plane wave expansion method as shown in Fig. 11. The band diagram of the photonic crystal along symmetry lines

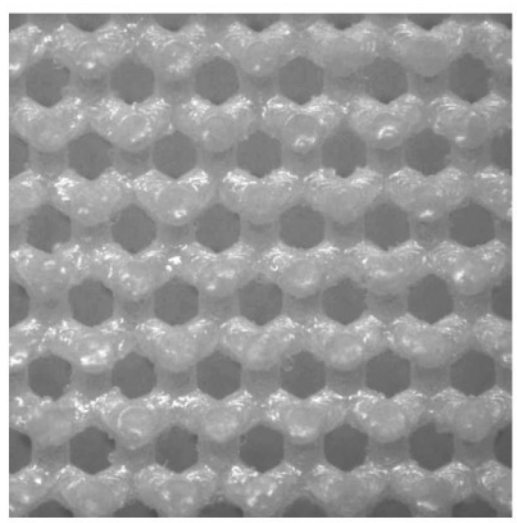

(100)

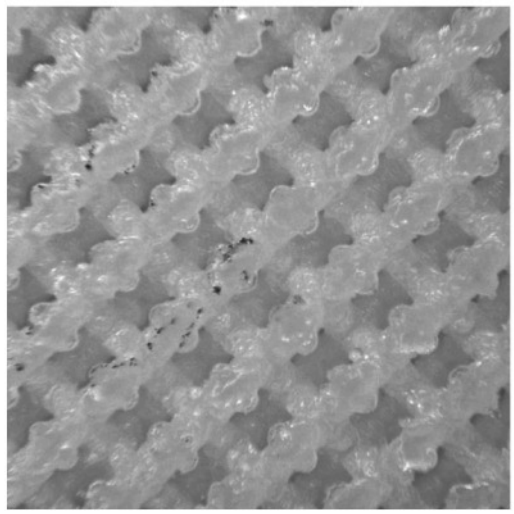

(110)

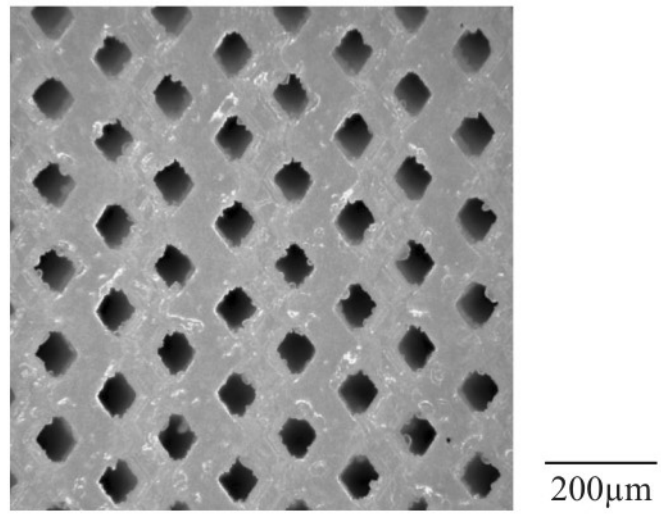

Fig. 9 Crystal planes of (111), (100) and (110) in alumina photonic crystals with the diamond structure fabricated by using the micro stereolithography. Sintering density of alumina lattices is $98.5 \%$. Dielectric constant of the lattice is 10 . 

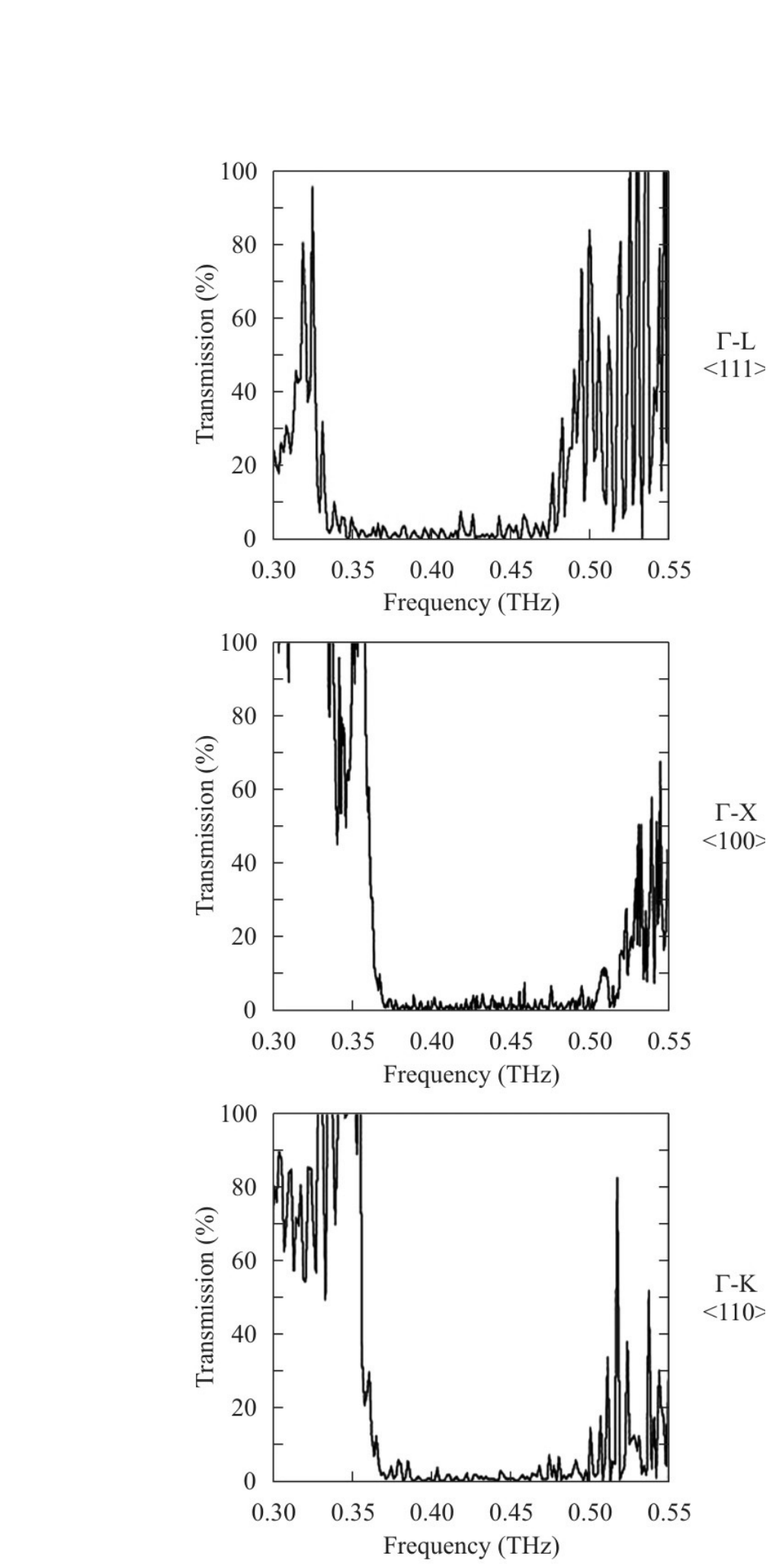

Fig. 10 Terahertz wave Attenuations of transmission amplitude for $\Gamma-\mathrm{L}$ $<111>, \Gamma-\mathrm{X}<100>$ and $\Gamma-\mathrm{K}<110>$ directions in the alumina photonic crystal with the diamond structure by using terahertz time domain spectroscope.

in the Brillouin zone is drawn theoretically by the PWE method. The opened circles mean the higher and lower edges of the measured band gaps. These frequency ranges of opaque regions corresponded to the calculation. According to the photonic band dia-

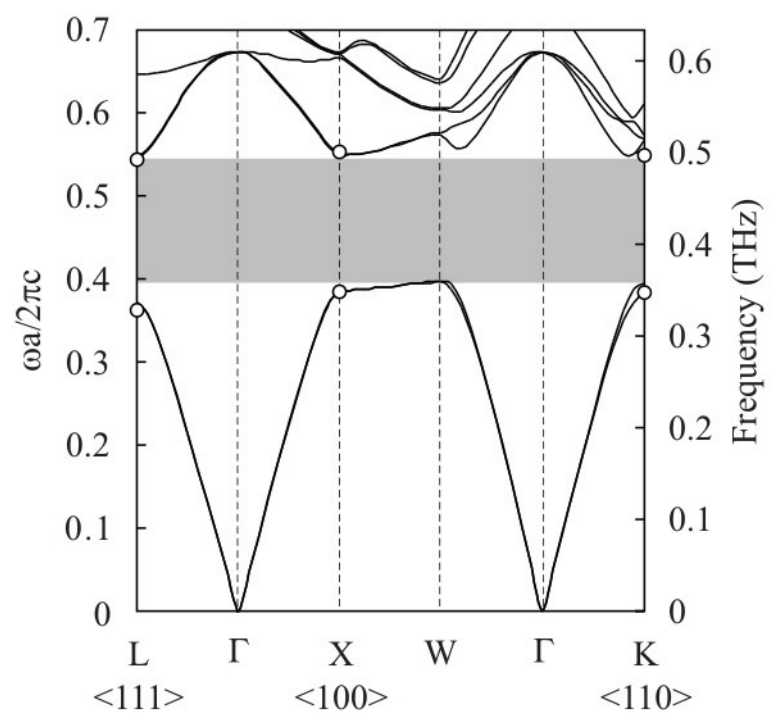

Fig. 11 A photonic band diagram calculated by plane wave expansion (PWE) method. Opened circles are measured edge frequencies of photonic band gaps. The dielectric constant of the lattice was 10. The frequency range with gray color indicates the perfect band gap in common for all directions.

gram, it was demonstrated that a complete photonic band gap opened between 0.35 and $0.49 \mathrm{THz}$. When a gap is formed, there are two types of the standing wave modes with the wavelength corresponding to periodicity of the dielectric lattices at the frequencies of the each band edges as shown in Fig. 1. The lower frequency mode concentrates the wave energy in the dielectric region, whereas the higher frequency mode concentrates in the air region.

\section{Point Defect of Air Cavity}

A diamond structure introduced by an air cubic defect with the same dimension as the unit cell is Fig. 12. The transmission spectrum along the $\Gamma-\mathrm{X}$ $<100>$ direction is shown in Fig. 13. Two peaks were observed in the band gap at the frequencies 0.42 and $0.46 \mathrm{THz}$, respectively. Measured peak frequencies were compared with the simulation by the TLM method as seen in Fig. 14. They were in good agreement with the simulation. The first peak in Fig. 13 was named mode $\mathrm{A}$, while the second one mode $\mathrm{B}$. The electric field distributions of these modes were simulated by the TLM method. Fig. 15 (a) and (b) show cross sectional images of the distributions. In the images, the red area indicates that the electric field intensity is high, whereas blue and green area indicates it is low. Thus, it was considered that the mode A concentrated the oscillation energy of a half wavelength with an antinode in the cube. Also, the 


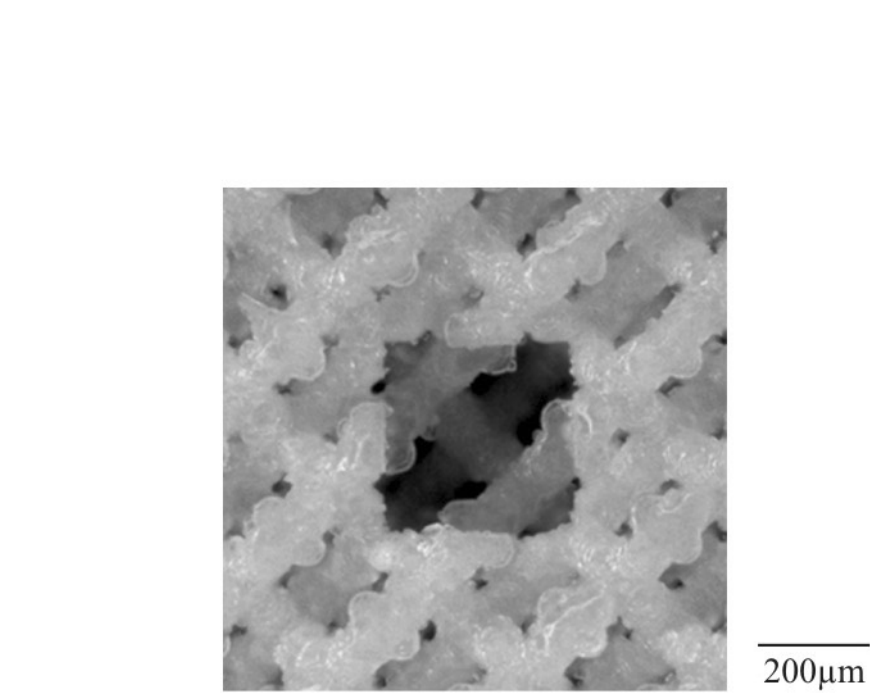

Fig. 12 A alumina lattice with the diamond structure introduced by an air cubic defect. The edge length of the air cavity is same size of the lattice constant.

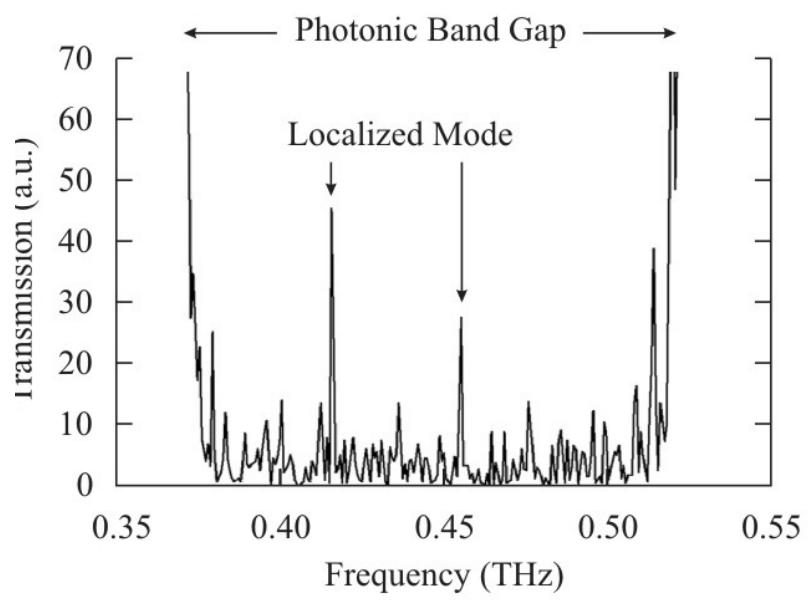

Fig. $13 \mathrm{~A}$ transmission spectrum of a defect introduced structure as a function of frequency. Two localized modes of transmission peaks are formed in the photonic band gap.

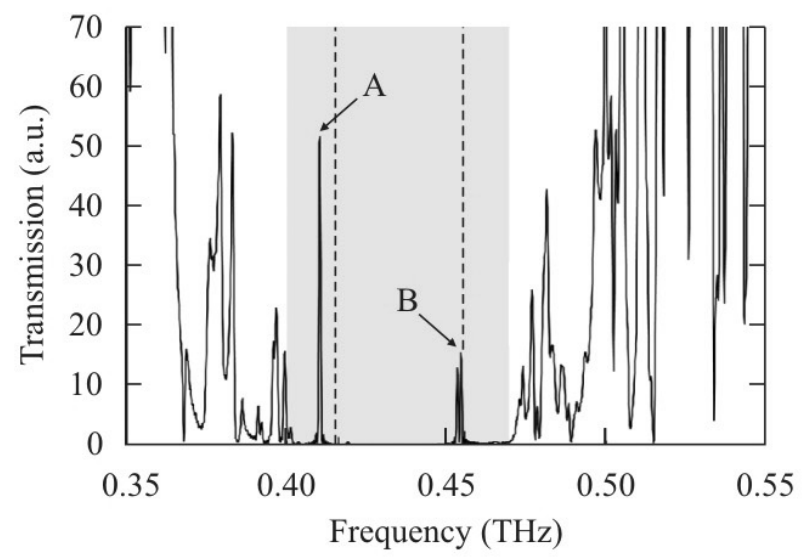

Fig. 14 The simulated spectrum of the defect introduced structure as a function of frequency. Dashed lines are frequencies of measured transmission peaks. The frequency range with gray color indicates the perfect band gap in common for all crystal directions.

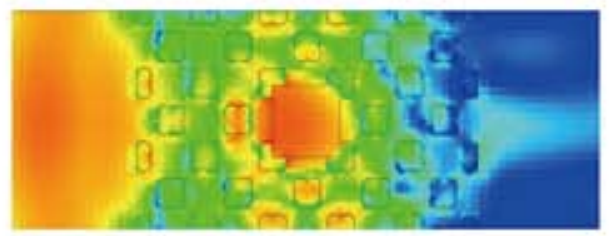

(a) Mode $\mathrm{A}$

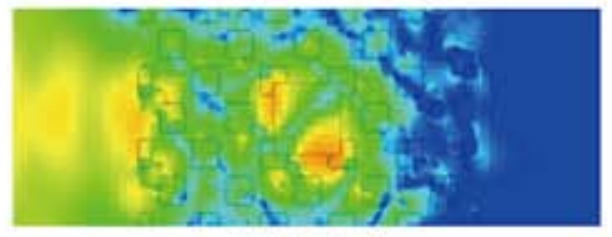

(b) Mode B

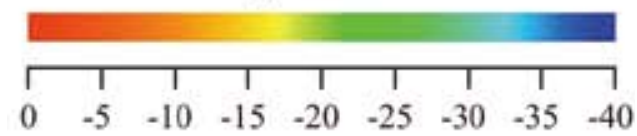

Electric Field Intensity [dB]

Fig. 15 Electric field distributions of localized mode A (a) and mode B (b) simulated by transmission line modeling (TLM) method of a finite difference time domain (FDTD).

mode B concentrated the energy of a half wavelength on the sides of the cube with a node in the cube. Therefore, it was confirmed that the defect introduced structure localized terahertz waves.

\section{Plane Defect of Twinned Lattices}

Twinned diamond structure composed of the mirror symmetric alumina lattices is shown in Fig 16. The plane defect forms parallel to the (100) crystal plane. The transmission spectrum for the $\Gamma-\mathrm{X}<100>$ crystal direction of the twinned diamond structure is shown in Fig. 17. The localized mode forms in the photonic band gap. At the transmission peak of 0.41 $\mathrm{THz}$ in frequency, the incident terahertz wave localized in the plane defect, and the amplified wave propagated to the other side of the crystal sample. The measured band gap region and the peak frequency of the localized mode were compared with the calculated spectrum by the TLM method as shown in Fig. 18. They were in good agreement. Subsequently, the electric field distribution in the twinned diamond lattices was simulated. Fig. 19 shows cross sectional images of the distributions. Incident terahertz wave is resonate and localized in the plane defect region between the twinned diamond lattices. The amplified electromagnetic wave by multiple reflections can transmit through the photonic crystal. Therefore, the transmission peak will be formed in the band gap. The three dimensional photonic band gap structure 


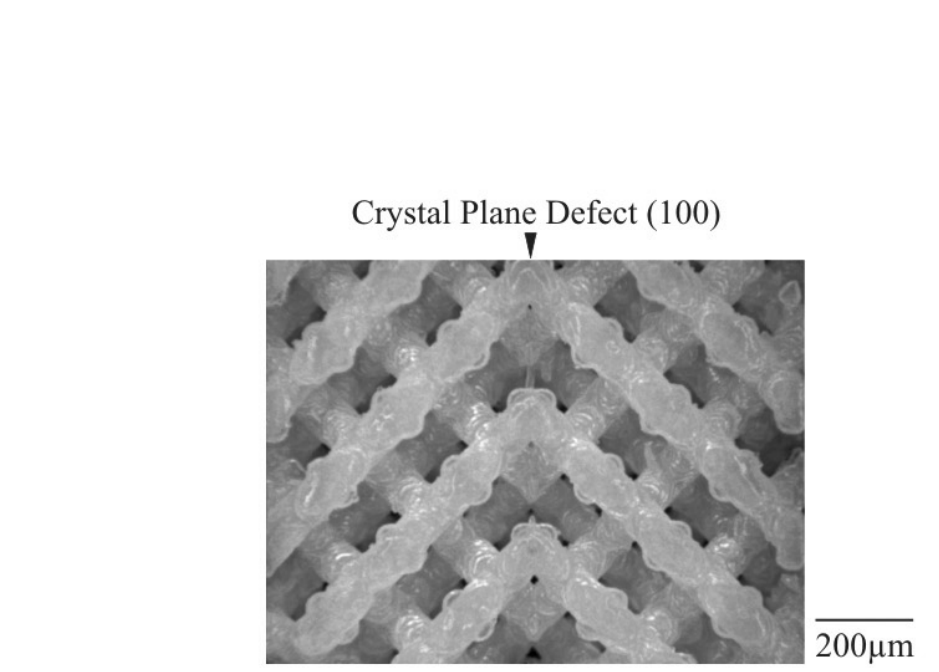

Fig. 16 A modified photonic crystal introduced by a plane defect parallel to (100) layers between twinned diamond lattices with a mirror symmetric arrangement for $\Gamma-\mathrm{X}<100>$ direction.

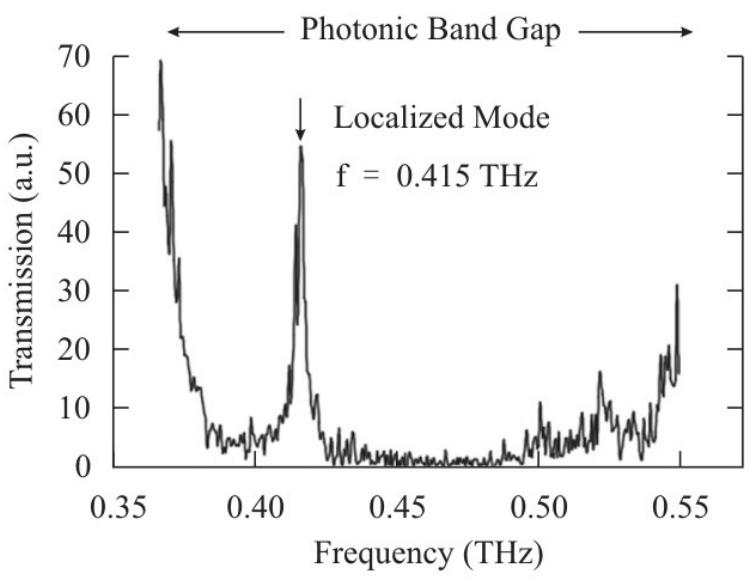

Fig. 17 The localized mode in the photonic band gap formed by the twinned diamond structure composed of the alumina lattices.

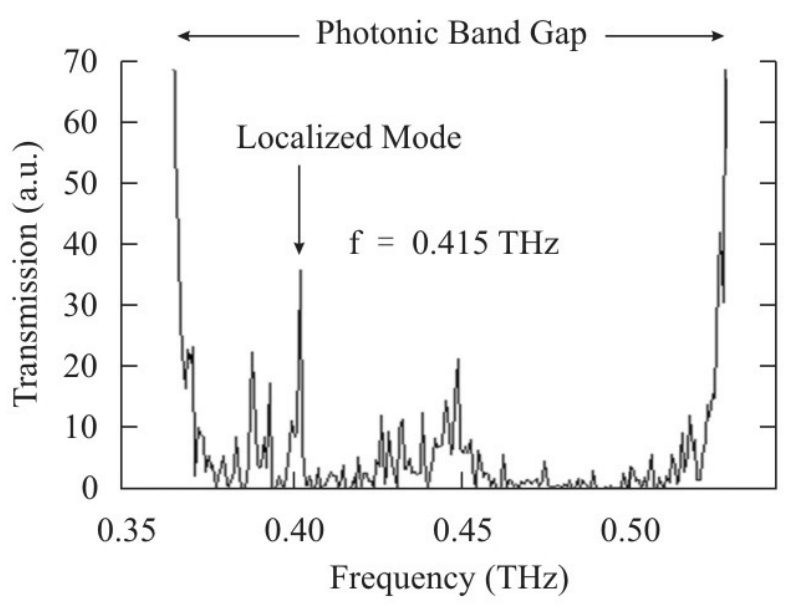

Fig. 18 The localized mode of the transmission peak in the photonic band gap formed by the twinned diamond structure. The perfect dielectric lattice forms the band gap to prevent the electromagnetic wave propagations, and the structural defect forms the localized mode to permit the transmission.

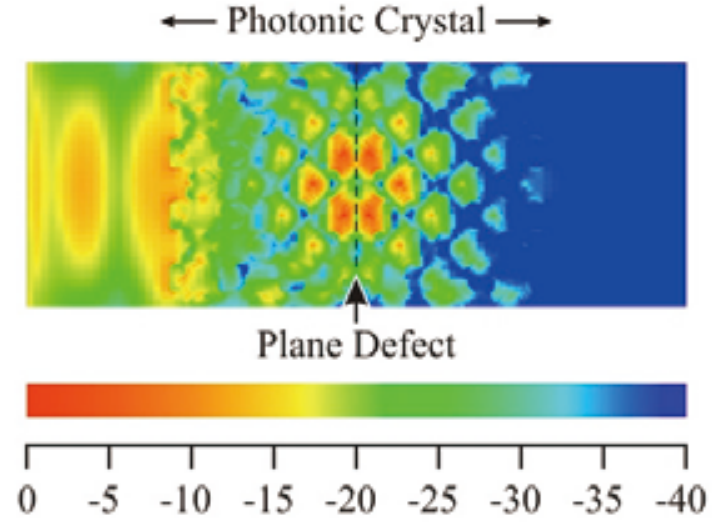

Electric Field Intensity [dB]

Fig. 19 The intensity distribution of electric field in the twinned diamond structure calculated by the TLM method. Incident terahertz wave are localized by multiple reflections in the plane defect between two dielectric lattice structures.

to form the localized mode can be applied to the terahertz wave filters.

\section{Terahertz Wave Beam Emitter}

A modified diamond photonic crystal with the plane defect between the twinned lattice structures is shown in Fig. 20. The lattice structures of one and two periods were arranged on the right and left side of the plane defect, respectively. The incident direction of the terahertz wave was from the left to the right. In the measured transmission spectrum, one localized mode peak was observed in the band gap at the frequencies of $0.41 \mathrm{THz}$. The measured

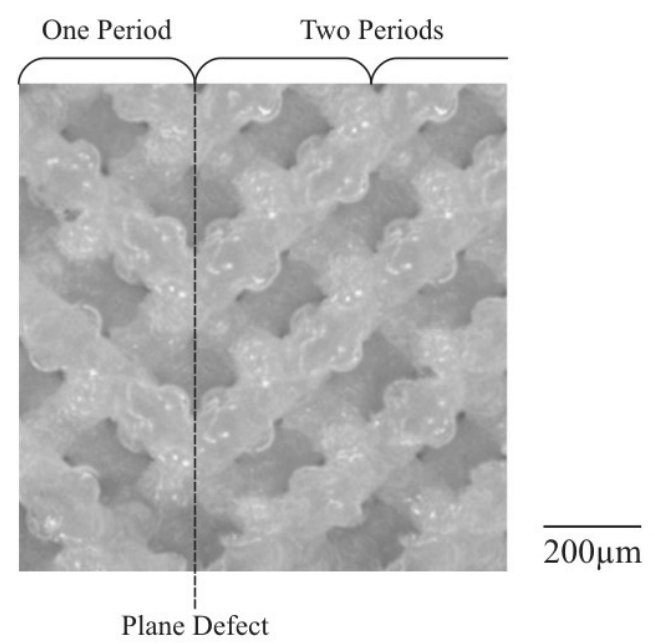

Fig. 20 An asymmetric twinned diamond structure. One and two periods of diamond lattices were formed on the left and right sides of the plane defect, respectively. 


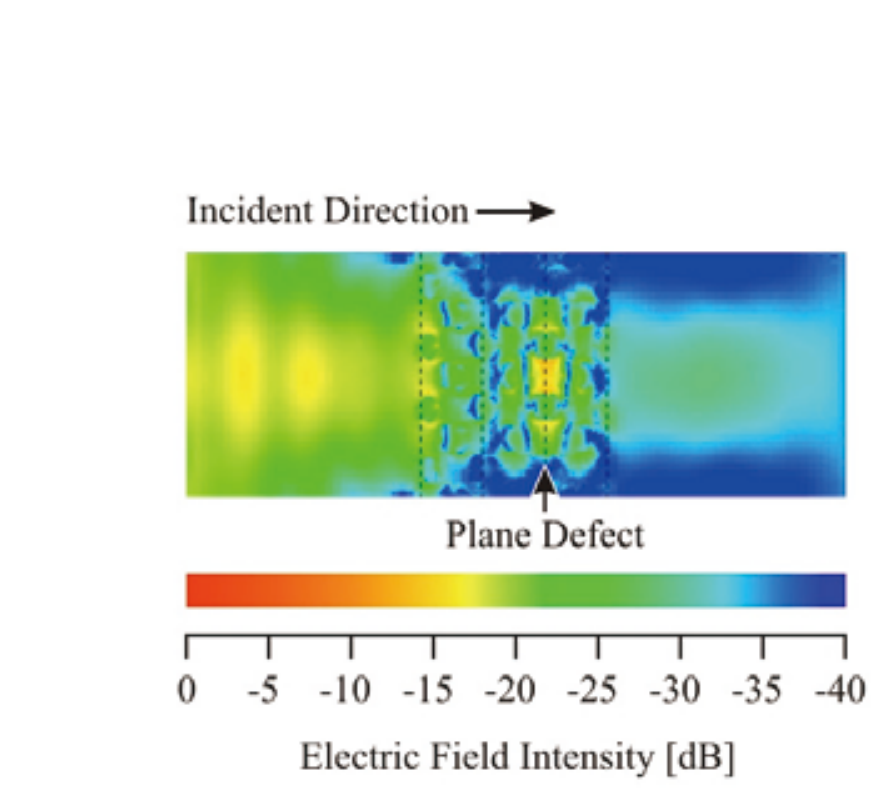

Fig. 21 The cross sectional image of the electric field intensity in the asymmetric twinned diamond structure calculated by the TLM method. The incident terahertz wave can transmit and propagate through the twinned lattice structure form the left to right side as an amplified plane wave.

band gap region and peak frequency of the localized mode were compared with calculations by the TLM method. They had good agreement. Subsequently, the electric field distribution in the twinned diamond lattices was simulated. Fig. 21 shows cross sectional images of the distributions. Incident terahertz wave is resonate and localized in the plane defect region. The amplified electromagnetic wave by multiple reflections can transmit preferentially for the right direction through the diamond lattice with only one period of the diffraction lattices. On the right side of the sample, the radiation pattern shows the plane wave expansion. The micro photonic crystal with the twinned ceramic lattice of the diamond structure can be applied to a terahertz beam emitters.

\section{Conclusion}

Three dimensional micrometer order photonic crystals with a diamond structure composed of acrylic resin including alumina nanoparticles at 40 vol. \% by using micro stereolithography a structural joining process. By the careful optimization of process parameters regarding dewaxing and sintering, dense alumina micro lattice structures were fabricated successfully. The sintered photonic crystal of alumina formed a complete band gap at the terahertz region from 0.35 to $0.49 \mathrm{THz}$. Localized modes were obtained by introducing a point defect of air cubic cavity and a plane defect between twinned diamond structures, which were in good agreements with the simulation by TLM method. It is expected that these three dimensional photonic band gap structures can be applied to control terahertz waves.

\section{Acknowledgments}

This study was supported by Priority Assistance for the Formation of Worldwide Renowned Centers of Research - The Global COE Program (Project: Center of Excellence for Advanced Structural and Functional Materials Design) from the Ministry of Education, Culture, Sports, Science and Technology (MEXT), Japan.

\section{References}

1) Ohtaka, K. (1979):Energy bang of photons and low energy photon diffraction” , Physical Review B, Vol. 19, No. 10, pp.5057-5067.

2) Yablonovitch, E. (1987):Inhabited Spontaneous Emission in Solid-state Physics and Electronics, Physical Review Letter, Vol. 58, No. 20, pp.2059-2062.

3) John, S. (1987):Strong Localization of Photons in Certain Disordered Dielectric Superlattices, Physical Review Letter, Vol. 58, No. 23, pp.2486-2489.

4) Temelkuran, B., Bayindir, M., Ozbay, E., Biswas, R., Sigalas, M., Tuttle, G. and Ho, K. (2000):Photonic Crystal-based Resonant Antenna with Very High Directivity, Journal of Applied Physics, Vol. 87, No. 1, pp.603-605.

5) Soukoulis, C. (1996): "Photonic Band Gap Materials" , Kluwer Academic Publsher, Netherlands,.

6) Noda, S., Yamamoto, N., Kobayashi, H., Okano, M. and Tomoda, K. (1999):Optical properties of threedimensional photonic crystals based on III-V semiconductors at infrared to near-infrared wavelengths, Applied Physics Letters, Vol. 75, No. 16, pp.905-907.

7) Kirihara, S., Takeda, M., Sakoda, K. and Miyamoto, Y. (2002): Control of Microwave Emission from Electromagnetic Crystals by Lattice Modifications, Solid State Communications, Vol. 124, No. 4, pp.135-139.

8) Kanehira, S., Kirihara, S. and Miyamoto, Y. (2005): Fabrication of $\mathrm{TiO}_{2}-\mathrm{SiO}_{2}$ Photonic Crystals with Diamond Structure, Journal of the American Ceramic Society, Vol. 88, No. 6, pp.1461-1464.

9) Ho, K., Chan, C. and Soukoulis, C. (1990): Existence of a Photonic Gap in Periodic Dielectric Structures, Physical Review Letter, Vol. 65, No. 25, pp.3152-3165.

10) Kirihara, S., Miyamoto, Y., Takenaga, K., Takeda, M. and Kajiyama, K. (2002): Fabrication of Electromagnetic Crystals with a Complete Diamond Structure by Stereolithography, Solid State Communications, Vol. 121, No. 8, pp.435-439.

11) Chen, W., Kirihara, S. and Miyamoto, Y. (2007): Fabrication and Measurement of Micro Three-Dimensional Photonic Crystals of $\mathrm{SiO}_{2}$ Ceramic for Terahertz Wave Applications, Journal of the American Ceramic Society, Vol. 90, No. 7, pp.2078-2081. 
120 Chen, W., Kirihara, S. and Miyamoto, Y. (2007): ThreeKOdimensional Microphotonic Crystals of $\mathrm{ZrO}_{2}$ Toughened $\mathrm{Al}_{2} \mathrm{O}_{3}$ for Terahertz wave applications, Applied Physics Letter, Vol. 91, No. 15, 153507-1-3.

13) Chen, W., Kirihara, S. and Miyamoto, Y. (2007): Fabrication of Three-Dimensional Micro Photonic Crystals of Resin-Incorporating $\mathrm{TiO}_{2}$ Particles and their Terahertz Wave Properties, Journal of the American Ceramic Society, Vol. 90, No. 1, pp.92-96.

14) Chen, W., Kirihara, S. and Miyamoto, Y. (2008): Static Tuning Band Gaps of Three-dimensional Photonic Crystals in Subterahertz Frequencies, Applied Physics Letters, Vol. 92, pp.183504-1-3.

15) Kanaoka, H., Kirihara, S. and Miyamoto, Y. (2008): Terahertz Wave Properties of Alumina Microphotonic Crystals with a Diamond Structure, Journal of Materials Research Vol. 23, No. 4, pp.1036-1041.

16) Miyamoto, Y., Kanaoka. H. and Kirihara, S. (2008): Terahertz Wave Localization at a Three-dimensional Ceramic Fractal Cavity in Photonic Crystals, Journal of Applied Physics, Vol. 103, pp.103106-1-5.

17) Takano, H., Song, B., Asano, T. and Noda, S. (2005): Highly Efficient in-Plane Channel Drop Filter in a Two-Dimensional Heterophotonic Crystal, Applied Physics Letters, Vol. 86, No. 24, pp.241101-1-3.

18) Kirihara, S. and Miyamoto, Y. (2009): Terahertz Wave Control Using Ceramic Photonic Crystals with Diamond Structure Including Plane Defects Fabricated by Micro-stereolithography, The International Journal of Applied Ceramic Technology, Vol. 6, No. 1, pp.41-44.

19) Kirihara, S., Niki, T. and Kaneko, M., "Terahertz Wave Behaviors in Ceramic and Metal Structures Fabricated by Spatial Joining of Micro-stereolithography, Journal of Physics, in printing.

20) Kirihara, S., Niki, T. and Kaneko, M., "Three-dimensional Material Tectonics for Electromagnetic Wave Control by Using Micoro-stereolithography”, Ferroelectrics, in printing.

21) Kirihara, S., Tsutsumi, K. and Miyamoto, Y., "Localization Behavior of Microwaves in Three-dimensional Menger Sponge Fractals Fabricated from Metallodielectric $\mathrm{Cu} /$ polyester Media”, Science of Advanced Materials, in printing.

22) Van Exter, M., Fattinger, C. and Grischkowsky, D. (1989): Terahertz Time-domain Spectroscopy of Water
Vapor, Optics Letters Vol. 14, Iss. 20, pp.1128-1130.

23) Clery, D. (2002): Brainstorming Their Way to an Imaging Revolution” , Science, Vol. 297, pp.761- 763.

24) Kawase K., Ogawa, Y., Watanabe, Y. and Inoue, H. (2003): Non-destructive Terahertz Imaging of Illicit Drugs Using Spectral Fingerprints, Optics Express, Vol. 11, Iss. 20, pp.2549-2554.

25) Woodward, R., Wallace, V., Arnone, D., Linfield, E. and Pepper, M. (2003): Terahertz Pulsed Imaging of Skin Cancer in the Time and Frequency Domain, Journal of Biological Physics, Vol. 29, No. 2-3, pp.257-259.

26) Wallace, V., Fitzgerald, A., Shankar, S. and Flanagan, N. (2004): Terahertz Pulsed Imaging of Basal Cell Carcinoma ex Vivo and in Vivo, The British Journal of Dermatology, Vol. 151, No. 2, pp.424-432.

27) Oyama, Y., Zhen. L., Tanabe, T. and Kagaya, M. (2008): Sub-Terahertz Imaging of Defects in Building Blocks, NDT\&E International, Vol. 42, No. 1, pp.28-33.

28) Haus, J. (1994): A brief review of theoretical results for photonic band structures, Journal of Modern Optics, Vol. 41, No. 2, pp.195-207.

29) Kawakami, S. (2002): "Photonic Crystals" , CMC, Tokyo,.

30) Noda, S. (2000): Three-dimensional photonic crystals operating at optical wavelength region, Physica B, Vol. 279, No. 1-3, pp.142-149.

31) Baba, T. and Fukuya, N. (2001): Light propagation characteristics of defects waveguides in a photonic crystal slab, Photonic Crystals and Light Localization (edited M. Soukoulis), Kluwer Academic Publishers, Netherlands,

32) Kosaka, H., Kawashima, T., Tomita, A., Notomi, M., Tamamura, T., Sato, T. and Kawakami, S. (1999): Photonic crystals for micro wave circuits using wavelength-dependent angular beam steering, Applied Physics Letter, Vol. 74, No. 8, pp.1370-1378.

33) Cregan, R., Mangan, B., Night, J., Birks, T., P. Russell, S., Roberts, P. and Allan, D. (1999): Singlemode photonic bandgap guidance of light in air, Science, Vol. 285, No. 5433, pp.1537-1539, September,.

34) Vos, W., Sprik, R., Blaaderen, A., Imhof, A., Lagendijk, A. and Wegdam, G. (1996): Strong effects of photonic band structures on the diffusion of colloidal crystals, Physical Review B, Vol. 53, No. 24, pp.16231-16235. 


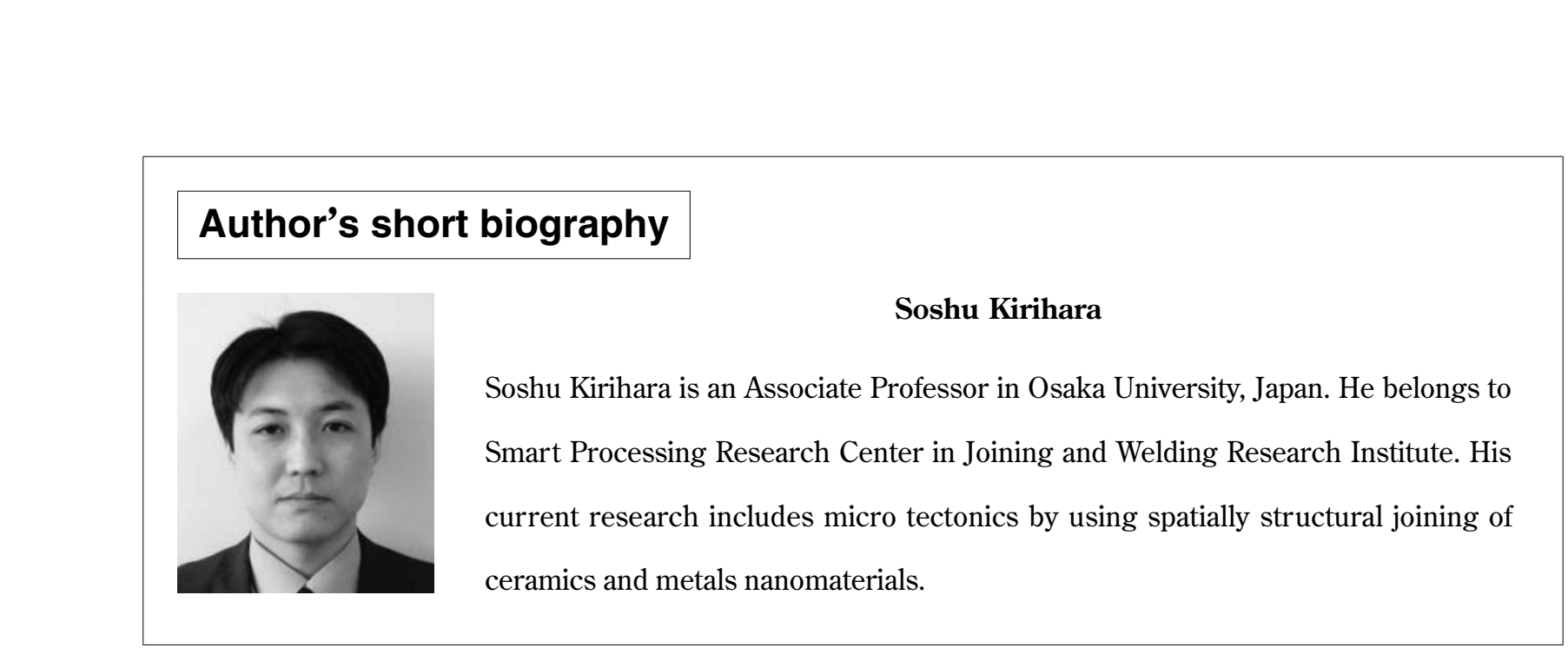

